Revue internationale P.M.E.

Économie et gestion de la petite et moyenne entreprise

\title{
Embauche et gestion des premiers employés d'une petite entreprise
}

\section{Kirk Neiswander, Barbara J. Bird et}

Volume 3, numéro 1, 1990

URI : https://id.erudit.org/iderudit/1007943ar

DOI : https://doi.org/10.7202/1007943ar

Aller au sommaire du numéro

Éditeur(s)

Presses de l’Université du Québec

ISSN

0776-5436 (imprimé)

1918-9699 (numérique)

Découvrir la revue

Citer cet article

Neiswander, K., Bird, B. J. \& (1990). Embauche et gestion des premiers employés d'une petite entreprise. Revue internationale P.M.E., 3(1), 7-25.

https://doi.org/10.7202/1007943ar
Résumé de l'article

Cette étude traite de la façon dont 52 entrepreneurs, à la tête d'entreprises d'âge et de secteurs d'industrie différents, ont choisi leurs premiers employés. Elle s'intéresse particulièrement aux cinq premiers employés et gestionnaires entrés en fonction et démontre que les entrepreneurs ont tendance à confier aux premiers arrivés un plus grand éventail de responsabilités qu'aux employés engagés ultérieurement. A l'embauche des directeurs, la priorité est donnée à la gestion des opérations, aux ventes et à l'administration; tandis que le poste de directeur du personnel et des ressources humaines est relégué au second plan et le dernier comblé. Il semble que le secteur de l'industrie, l'âge de l'entreprise et les antécédantsde l'entrepreneur influencent la gestion des ressources humaines de départ. L'étude traite également d'aspects tels le développement de l'«esprit d'entreprise», la délégation des responsabilités et la rémunération, ainsi que des changements de la structure organisationnelle entraînés par l'expansion de l'entreprise. 


\title{
Embauche et gestion des premiers employés d'une petite entreprise
}

\author{
D. Kirk NEISWANDER' \\ Barbara J. BIRD \\ Peter L. YOUNG \\ Case Western University
}

\begin{abstract}
RÉSUMÉ
Cette étude traite de la façon dont 52 entrepreneurs, à la tête d'entreprises d'âge et de secteurs d'industrie différents, ont choisi leurs premiers employés. Elle s'intéresse particulièrement aux cinq premiers employés et gestionnaires entrés en fonction et démontre que les entrepreneurs ont tendance à confier aux premiers arrivés un plus grand éventail de responsabilités qu'aux employés engagés ultérieurement. A l'embauche des directeurs, la priorité est donnée à la gestion des opérations, aux ventes et à l'administration; tandis que le poste de directeur du personnel et des ressources humaines estrelégué au second plan et le dernier comblé. Il semble que le secteur de l'industrie, l'âge de l'entreprise et les antécédants de l'entrepreneur influencent la gestion des ressources humaines de départ. L'étude traite également d'aspects tels le développement de l' "esprit d'entreprise", la délégation des responsabilités et la rémunération, ainsi que des changements de la structure organisationnelle entraînés par l'expansion de l'entreprise.
\end{abstract}

\section{ABSTRACT}

The early hiring practices of 52 entrepreneurs in different industries and of different ages are examined. With an emphasis on the first five employees and the earliest managers hired, this study finds that earlier employees tend to be given a wider range of responsabilities than later hires. As managers are hired, the focus is on operations, administration and sales, with the manager of personnel and human resources being the least important and last hired. Issues of finding and motivating "entrepreneurial spririt", delegation, and compensation are discussed as are some of the changes in organizational structure that occur with increased size. Industry, age of firm, and background of the entrepreneur appear to influence early human resource practices.

* D. Kirk Neiswander est directeur du programme en entrepreneuriat de Weatherhead School of Management à l'Université Case Western Reserve. Barbara J. Bird, est professeur à cette université. Elle a publié de nombreux articles et récemment un ouvrage intitulé «Entrepreneurial behavior». Peter L. Young est aussi professeur à l'Université Case Western Reserve. Adresse : The Weathearhead School of Management, Case Western Reserve University, Cleveland, Ohio 44106, U.S.A. 


\section{RESUMEN}

Este esiudio trata sobre la manera por medio de la cual 52 empresarios, dirigentes de empresas antigüas y de diferentes sectores industriales, han seleccionado sus primeros empleados. Se acuerda asi entonces un interés particular a los cinco primeros empleados y gestionarios que entraron en funcion y se demuestra que los empresarios tienen tendencia a confiar a los recien llegados un pliego mas grande de responsabilidades que al personal que se emplea ulteriormente. Se mentiona tambien que durante la seleccion de los de los directores, se da prioridad a la gestion de operaciones, a las ventas y a la administracion, mientras que el cargo de director de personal se llena en ultimo lugar. Parece entonces que el sector industrial, la antigüedad de la empresa y los antecedentes del empresario influyen sobre la gestion de los recursos humanos desde un comienzo. Para terminar, el estudio trata iqualmente sobre aspectos tales como el desarrollo del eespiritu de empresan, la delegacion de responsabilidades y la remuneracion como tambien sobre los cambios de la estructura de organizacion ocasionados por la expansion de la empresa. 


\section{Introduction}

Quels sont les conditions nécessaires au succès d'une nouvelle entreprise. Il semble que trois éléments principaux soient toujours présents :

1 Un produit ou un service pour lequel les gens sont prêts à payer.

2 du capital financier,

3 et des ressources humaines (une bonne gestion) pour démarrer l'entreprise.

De nombreuses études ont dressé le portrait physique, émotionnel, psychologique et biographique du fondateur d'une entreprise. Nombreuses également sont les enquêtes portant sur les sources de financement potientielles et la façon de financer une nouvelle entreprise. Toutefois, très peu d'études ont été menées sur la manière dont l'entrepreneur se dote de ses atouts les plus fondamentaux : ses premiers employés. Ce sont eux pourtant qui auront l'impact le plus déterminant sur l'éventuelle prospérité de l'entreprise. Ils constitueront aussi le premier défi de management auquel l'entreprencur sera confronté. Tout au long de la vie d'une petite entreprise, les questions relatives aux employés seront la source des plus grands problèmes que devront affronter les gestionnaires (Lewis et alii).

Les premiers employés devront : s'adapter à l'entreprise et évoluer avec elle; être extrêmement productifs, développer leur esprit d'initiative; correspondre à l'image de l'entreprise; favoriser la réalisation du rêve du fondateur; tout en faisant preuve de créativité pour résoudre la multitude de problèmes rencontrés dans l'exercice de leurs fonctions.

Où l'entrepreneur déniche-t-il de tels employés ? Quel est l'importance de s'entourer dès le début d'une bonne équipe ? (Un des entrepreneurs interrogés dans ce sondage a dû congédier, en raison de leur incompétence, toutes les personnes qu'il avait initialement engagées pour ensuite recommencer et finalement réussir avec une entreprise du même type.

Ce projet de recherche a été entrepris pour analyser de plus près comment l'entrepreneur choisit ses premiers employés et saisir leur influence sur le succès de l'entreprise.

De plus, cette recherche veut également répondre aux questions suivantes :

- Quelles étaient les pratiques de recrutement et d'embauche lors de la sélection?

- Quel était le rôle de la première personne engagée et quelles étaient ses responsabilités?

- Dans quel ordre les postes de direction ont-ils été comblés ?

- Quelles ont été les plus importantes préoccupations relatives au personnel dans les premières années de l'entreprise? 
- Quelles étaient les méthodes de formation, de motivation, de rémunération et de délégation des responsabilités?

- Comment la croissance de l'entreprise a-t-elle influencé la structure organisationnelle?

- A quelles étapes du processus d'embauche d'un candidat une aide pourrait-elle être apportée à l'entrepreneur?

Nous croyons que les résultats de cette enquête pourraient fournir des renseignements précieux pour l'entrepreneur sur le point de fonder une nouvelle entreprise et s'avérer une référence très utile pour ceux qui sont déjà en affaires.

\section{Plan de recherche}

Nous avons mené une enquête auprès des propriétaires-fondateurs d'entreprises indépendantes pour recueillir des données sur les pratiques d'embauche au moment de la création de leur firme.

L'objectif de cette étude était de mieux comprendre la façon dont l'entrepreneur gère le recrutement de ses premiers employés et travaille avec eux. Alors qu'il existe quelques données sur l'importance des équipes internes dans les nouvelles entreprises (Martin, 1984), on ignore presque tout des liens spécifiques entre les étapes de la constitution d'une équipe et de l'ordre chronologique de ces étapes. Pour combler ces lacunes, nous avons préparé un sondage avec des questions ouvertes.

\section{$2.1 \quad$ Le sondage}

Il s'agit d'un questionnaire de sept pages comprenant 15 questions, accompagné d'une annexe de cinq pages et 12 questions additionnelles à titre de «sondage optionnel». Chaque questionnaire a été conçu pour requérir de 15 à 20 minutes à remplir. Nous avons de plus procédéà cinq tests préliminaires par le biais d'entrevues personnelles.

\subsection{L'échantillon}

L'échantillon de ce sondage regroupait 350 entreprises situées dans les limites de l'indicatif téléphonique régional 216 (nord-est de l'Ohio). Il a été constitué à partir des listes de l'Ohio Industrial directory de Harris, du 51000 Largest U.S. Corporations de Ward, du Crain's Cleveland Business, du «Financial Survey of Middle Market Publicly Held Companies in Northeast Ohio» (Alexander Grant \& Co.) du Inc. Magazine et du Million Dollar Directory de Dunn \& Bradstreet». Nous y avons ajouté d'autres entreprises à la suite de lectures d'articles de journaux et de contacts avec des gens d'affaires de la région. 


\section{$2.3 \quad$ Méthode}

Les questionnaires ont été postés, au cours de la dernière semaine de novembre 1986 avec une lettre de présentation d'une page identifiant les objectifs du sondage, l'expéditeur ainsi que les personnes et le type d'entreprises visés par l'enquête. Si le destinataire s'apercevait de son inéligibilité (i.e. qu'il n'avait pas participé à la création de l'entreprise ou que cette demière n'était pas de type individuel), il était invité à renvoyer le matériel de sondage à l'expéditeur avec une note explicative. Afin d'obtenir un taux de réponses maximal, nous avons communiqué à deux reprises par téléphone avec les personnes qui n'avaient pas retourné le questionnaire.

Parmi les 350 entreprises consultées, 52 étaient inéligibles et neuf ont refusé directement de participer. Les résultats publiés dans ce rapport sont établis à partir des 52 questionnaires remplis et valides (taux de réponse de 18\%) et des 38 questionnaires optionnels retournés (taux de réponse de 13\%).

Les résultats seront présentés selon la fréquence des réponses aux différents points du questionnaire. De plus, nous en avons réparti certains selon le secteur de l'industrie, la formation et l'expérience des entrepreneurs et l'âge des entreprises.

Dans une répartition par secteur industriel, on dénombre 27 entreprises manufacturières, 17 commerces de gros et détail et 19 entreprises de services, (certains entrepreneurs du secteur manufacturier ont également classé leur entreprise dans le secteur du commerce de gros et détail ou de services). Nous avons regroupé les entrepreneurs selon leur expérience professionnelle : 25 d'entre eux avaient des antécédents en gestion seulement et 23 autres possédaient une expérience de gestion et une expérience technique ou en ingénierie. Dans une répartition selon l'âge, les entreprises se sont distribuées de la façon suivante : 19 de moins de 10 ans, 17 entre 10 et 19 ans, et 15 de plus de 20 ans. L'âge moyen des entreprises était de 14,3 ans. Nous n'avons pas tenu compte, dans cette étude, de la valeur actualisée de l'argent dans le cas des plus vieilles firmes. De plus, notons que les fondateurs de ces dernières peuvent avoir éprouvé plus de difficutlés à se souvenir des premiers moments de leur entreprise.

\section{Sommaire des résultats}

\subsection{Quelles étaient les personnes interrogées ?}

Les entrepreneurs consultés étaient de sexe masculin et, dans l'ensemble, possédaient au moins le diplôme de premier cycle universitaire. En moyenne, leur entreprise avait 14 ans, un chiffre d'affaires de 19,3\$ millions en 1986 et employait environ 35 personnes. Pour la première année d'exploitation de leur entreprise, les 
dirigeants interrogés ont déclaré un revenu moyen de 465000 et sept employés. Le tableau 1 suivant présente la façon dont nous avons regroupé les personnes interrogées.

\subsection{Quelles étaient les pratiques de l'entrepreneur au moment du recrutement et de l'embauche de ses premiers employés ?}

Dans la plupart des cas, l'entrepreneur, n'ayant par les moyens de se payer le luxe d'un «service de sélection du personnel cadre», procède par essais et erreurs pour choisir ses premiers employés. Généralement, il engage des amis ou des membres de sa famille. La plupart des entrepreneurs considèrent que les meilleurs candidats leur sont recommandés par des relations d'affaires, plus particulièrement d'un secteur de l'industrie. Très souvent les premiers employés sont d'anciens collègues ou proviennent d'entreprises apparentées ou de la concurrence ${ }^{1}$.

Au moment de recruter les premiers membres du personnel administratif, les entrepreneurs ont analysé en moyenne 12,6 curriculum vitae, rencontré 4,5 candidats qualifiés, et ont généralement convoqué le futur employé à une deuxième, voire même à une troisième entrevue.

Tab. 1 Classification de l'échantillon en sous-groupes

\begin{tabular}{|l|c|c|c|c|}
\cline { 2 - 5 } \multicolumn{1}{c|}{} & $\begin{array}{c}\text { Secteur } \\
\text { manufacturier }\end{array}$ & $\begin{array}{c}\text { Secteur commercial } \\
\text { gros et détail }\end{array}$ & $\begin{array}{c}\text { Secteur } \\
\text { services }\end{array}$ & Total \\
\hline $\begin{array}{l}\text { Entrepreneurs avec } \\
\text { expérience de gestion }\end{array}$ & 12 & 5 & 8 & 25 \\
$\begin{array}{l}\text { Entrepreneurs avec } \\
\text { expérience technique } \\
\text { et de gestion }\end{array}$ & 15 & 3 & 5 & 23 \\
\hline TOTAL & 27 & 8 & 13 & $n=48$ \\
\hline $\begin{array}{l}\text { Age moyen de } \\
\text { l'entreprise (années) }\end{array}$ & 17,9 & 22,1 & 10,8 & 14,3 \\
\hline
\end{tabular}

- Quelques-uns de ces entrepreneurs nous ont indiqué qu'ils opéraient dans plus d'un secteur (i.e. manufacturier et des services). Plus loin dans cette recherche leurs réponses figurent dans les deux secteurs.

1 D'après les conclusions de la recherche : «The Origins of Successful Start-up Firms in Northeast Ohio», du D. Kirk Neiswander et de John M. Drollinger, 1986. 


\subsubsection{Les entrevues}

Lors de la première entrevue, les renseignements les plus pertinents pour l'entrepreneur concernaient les buts et objectifs du candidat et ses projets pour les cinq années qui viennent. L'expérience du candidat, son aptitude à résoudre des problèmes particuliers, son sens de l'initiative, son dynamisme, ses motivations et sa capacité d'apprendre et de devenir productif rapidement venaient en deuxième lieu, mais étaient encore considérés très importants. Au cours de la deuxième entrevue, on demandait généralement au candidat quelle autre contribution il pouvait apporter à l'entreprise et pourquoi il désirait travailler. Si les réponses du candidat étaient satisfaisantes, l'entrepreneur, dans son empressement à combler un poste le plus tôt possible, l'a souvent engagé sur-le-champ.

Il semble que certains points pouvant avoir des répercussions à long terme sur l'entreprise étaient négligés, entre autres : la «compatibilité» du candidat à la culture de l'entreprise et, bien que ce soit plus difficile à évaluer, sa capacité d'accéder à de nouveaux postes et de prendre en charge de nouvelles responsabilités. Dans l'ensemble, les premiers employés n'ont été engagés que pour satisfaire au besoin spécifique du moment.

Lorsqu'interrogés sur les plus importantes caractéristiques ou qualités que devraient posséder les premiers gestionnaires ou administrateurs potentiels, plus des deux tiers des entrepreneurs consultés ont énuméré les qualités suivantes : esprit d'entreprise, dynamisme, auto-motivation, initiative et goût de l'action. Les qualifications et l'expérience professionnelle sont les points qui retiennent le plus l'attention des entrepreneurs au moment de combler un poste. Le tiers des personnes interrogées ont mentionné avoir vérifié les références des candidats. Ceux qui ont satisfait aux conditions ont été engagés et les autres éliminés d'après l'intuition de l'entrepreneur et le comportement des candidats au cours du processus d'entrevues.

\subsection{Quel était le rôle de la première personne engagée et quelles étalent ses responsabilités ?}

Au moment de l'embauche du premier employé, le capital des deux tiers des entreprises consultées représentait moins de 25000 \$. Dans $75 \%$ des cas, l'entrepreneur avait investi ses fonds personnels (neuf des personnes interrogées ont financé à $100 \%$ leur entreprise), ce qui donne au lecteur une idée de l'approche conservatrice de celui-ci des dépenses qu'occasionne l'embauche d'un employé.

Dans 50\% des cas étudiés, le premier employé a été engagé au cours du mois où les premières ventes ont été réalisées avec, toutefois, des différences appréciables selon l'âge de la firme. Toutes les entreprises de moins de 20 ans avaient engagé un premier employé au moment de leur première vente ou avant. En ce qui concerne les entreprises de 20 ans ou plus, $50 \%$ d'entre elles ont engagé quelqu'un dans les six mois suivant leur première vente. Ce constat indique une attitude encore plus conservatrice des entrepreneurs devant l'embauche des premiers employés dans les années soixante que celle prévalant aujourd'hui. 


\subsubsection{Le premier employé}

La première personne engagée avait un poste regrougant, en moyenne, 2,3 fonctions différentes (i.e. qu'un employé pouvait avoir des responsabilités dans trois fonctions différentes tels le marketing, la production et la comptabilité). Au nombre des postes qui justifiaient le plus fréquemment l'embauche du premier employé figurent les ventes, la gestion des opérations (production), l'administration ou le secrétariat. Selon son rang d'arrivée, l'employé se voyait confier moins de fonctions que le précédent. A l'embauche de la cinquième personne, une seule fonction était assignée. En ce qui concerne les firmes créées dans les années soixante-dix et quatrevingt, une nette tendance semble également démontrer que plus de responsabilités sont confiées aux premiers employés comparativement aux entreprises plus anciennes où l'on engageait une personne pour occuper une seule fonction spécifique.

Comme on peut s'en douter, les entrepreneurs ayant une expérience technique ont tendance à confier à leurs premiers employés des postes techniques ou d'ingénierie. A leurs débuts, les firmes fondées dans les dix dernières années semblent surtout rechercher des gens pour assumer des responsabilités reliées aux ventes.

\subsubsection{Observations sur les cinq premiers employés}

- Il y a de fortes possibilités pour qu'un entrepreneur ayant une expérience technique engage, comme premier employé, une personne pour travailler aux opérations avant d'embaucher un employé de secrétariat. L'entrepreneur sans expérience technique fera plutôt le contraire.

- Les entrepreneurs du secteur manufacturier ont tendance à placer la totalité ou la plupart de leur cinq premiers employés dans les secteurs de production et d'opérations.

- On s'attendait de beaucoup à ce que les premiers employés assument des responsabilités dans le secteur des ventes.

- A partir du troisième employé, on tenait davantage compte du service de la comptabilité.

- Les entrepreneursà la tête d'entreprises fondées dans les dix dernières années ont engagé leurs premiers employés pour occuper des fonctions reliées aux ventes, alors que les fondateurs de firmes plus anciennes n'ont embauché au début que du personnel affecté aux opérations. Il semble donc que le rôle de l'entrepreneur directeur général soit passé graduellement de celui de vendeur principal à celui de gérant général des opérations internes, et que les nouvelles entreprises aient une approche plus axée sur le marketing.

\subsubsection{Recommandations pour l'embauche du premier employé}

Se remémorant leurs expériences passées, les entrepreneurs consultés pour cette enquête donnent les conseils suivants :

1 L'entrepreneur devrait engager une personne qui lui est compatible en termes de 
valeurs, principes, conscience profesionnelle, ambition et initiative. Il devrait faire en sorte de connaître le candidat personnellement.

2 L'entrepreneur devrait choisir un candidat avec une expérience pertinente au poste à combler et qui a déjà travaillé dans une petite entreprise. Il devrait éviter les périodes de formation coûteuses, le mettre au travail tout de suite et exiger immédiatement du rendement (bien sûr, il fera des erreurs, mais il apprendra plus rapidement «sur le tas»).

3 L'entrepreneur devrait embaucher le candidat qui, de part son intelligence et son éducation, peut apporter le plus à l'entreprise.

4 L'entrepreneur devrait sélectionner le candidat qui peut évoluer au rythme de la firme. Il devrait trouver une personne qui projette de rester au moins cinq ans avec l'entreprise et qui aime les défis, les risques et les satisfactions associées à une entreprise qui démarre.

\subsection{Dans quel ordre les postes de direction ont-lls été comblés ?}

Différents types d'entreprises ont diverses raisons pour engager des employés à des stades distincts de leur croissance. Les entrepreneurs semblent considérer le directeur de la gestion des opérations, le chef de bureau ou le directeur commercial comme l'un des trois employés les plus importants. Bien que les entrepreneurs engagent le directeur du marketing au cinquième rang et le directeur des achats au sixième rang, ils accordent au contrôleur, qui est embauché plus tard, une plus grande valeur. (Cela pourrait s'expliquer par la plus grande proportion d'entrepreneurs du secteur manufacturier dans l'échantillon.) Le graphique 1 nous permet de visualiser la valeur donnée à chaque poste et la période écoulée, depuis les débuts de l'exploitation, avant qu'il ne soit comblé.

\subsubsection{Observations}

- Le taux de croissance de la firme influencera les pratiques d'embauche et inversement.

- Les entreprises fondées il y a plus de vingt ans ont, dans l'ensemble, attendu plus de dix ans avant d'engager un contrôleur, tandis que les entreprises plus récentes l'ont fait au cours de leur deuxième ou troisième année d'existence.

- Environ la même proportion d'entrepreneurs sans expérience technique que d'entrepreneurs possédant une expérience technique ont engagé des gestionnaires techniciens ou ingénieurs au cours de la première année de leur entreprise.

- Les entrepreneurs techniciens ont engagé un directeur de la comptabilité ou des finances plus tôt que les entrepreneurs possédant une expérience de gestion ne l'ont fait.

- Les entrepreneurs techniciens ont semblé moins concernés par l'embauche d'un chef de bureau.

- Les entreprises plus jeunes ont eu besoin d'un chef de bureau au cours de leur première année d'exploitation. 
- Les entreprises plus jeunes ont employé un directeur des achats beaucoup plus tôt que les entreprises plus vieilles ne l'ont fait.

\subsection{Quels ont été les plus importantes préoccupations relatives au personnel dans les premlères années de l'entreprise?}

Dans la liste suivante, les entrepreneurs énumèrent les préoccupations relatives au personnel par ordre décroissant d'importance :

1 L'embauche d'un employé

2 Le moral

3 La motivation

4 La productivité

5 La rotation du personnel

6 La formation

7 La rémunération

Graph. 1 Importance et ordre d'embauche des gestionnaires pour chacun des postes de responsabilité suivants

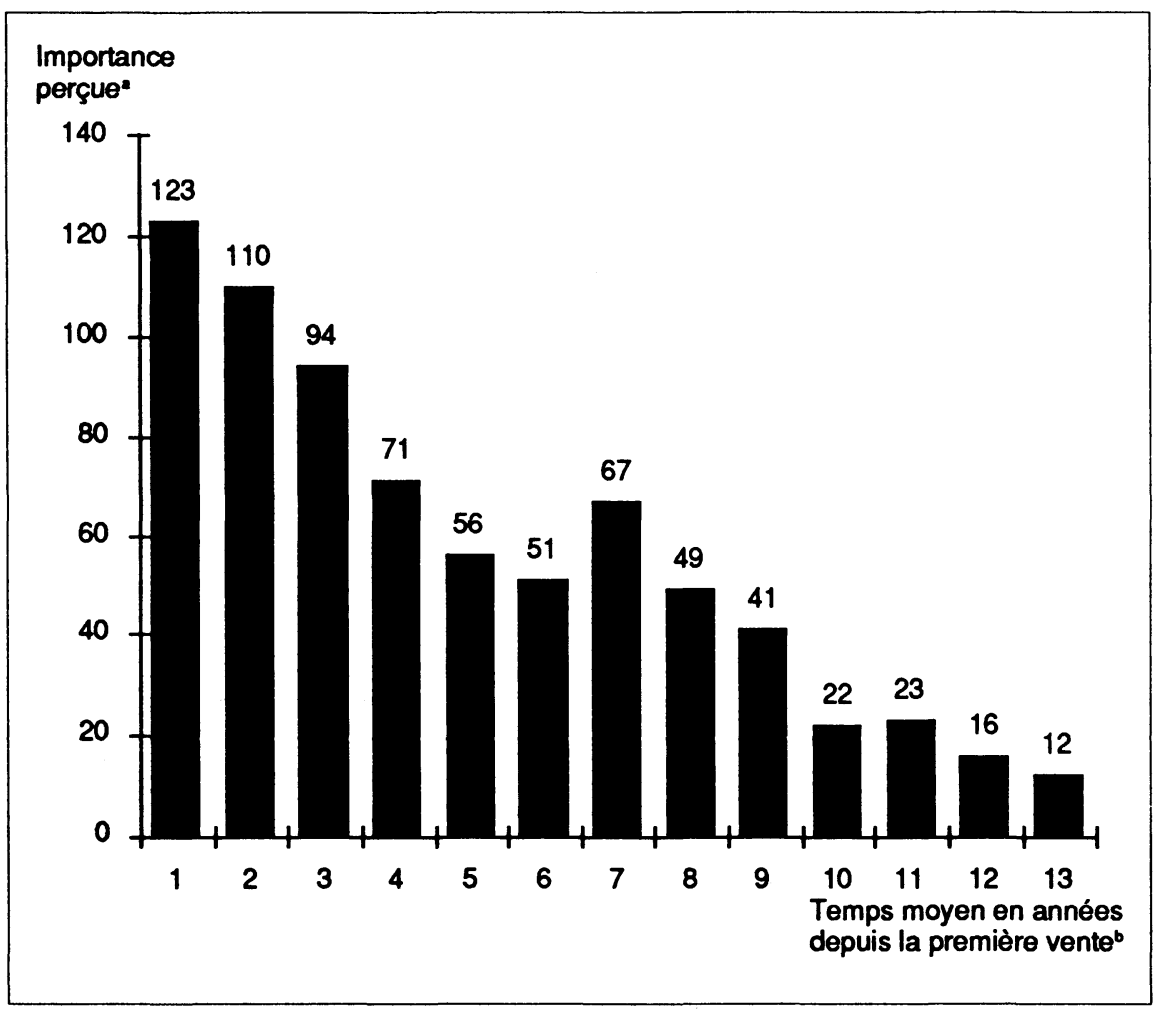


a Nous avons calculé la cote de l'importance perçue de la façon suivante : chaque personne interrogée a établi l'ordre d'embauche des gestionnaires pour 13 postes de responsabilités. Chaque premier vote a reçu 5 points, chaque deuxième vote 4 points et ainsi de suite jusqu'au cinquième vote (1 point). Donc, l'importance perçue était fonction du nombre de votes et du rang dans lequel les votes ont été accordés.

b Etant donné qu'il s'agit d'une moyenne, la représentation graphique de l'ordre d'embauche est fidèle mais l'année de la première vente au cours de laquelle le poste a effectivement été comblé ne l'est pas nécessairement.

L'embauche était, et de loin, la préoccupation principale de tous les sondés, peu importe le secteur de l'industrie, l'âge de la firme et les antécédents professionnels des entrepreneurs. Les entrepreneurs à la tête d'entreprises fondées dans les années quatre-vingt semblent accorder plus d'importance à la formation de leurs employés. La rémunération apparaissait comme le dernier des soucis des entrepreneurs. Cela n'a rien d'étonnant étant donné que les entrepreneurs font souvent de longues journées de travail pour de petits salaires, surtout pendant les premières années de la firme, et qu'ils s'attendent à la même chose de leurs employés.

\subsection{Quelles étaient les méthodes de formation, de motivation, de rémunération et de délégation des responsabilités ?}

\subsubsection{La formation}

Au cours des premières années d'opération de leur firme, les entrepreneurs ne pouvaient se permetre le luxe de la formation de leurs employés et seulement $17,6 \%$ d'entre eux mentionnent des «programmes de développement du niveau de compétence des employés». Les personnes engagées étaient déjà formées ou pouvaient apprendre rapidement «sur le tas» et être productives dès le départ.

\subsubsection{La motivation}

Nombre d'entrepreneurs constatent que le manque «d'esprit entrepreneurial» de leurs employés constitue un obstacle majeurà la croissance de leur entreprise. Qui plus est, ils estiment que cet «esprit entrepreneurial» ne s'acquiert pas facilement; les employés l'ont ou pas. L'entrepreneur doit stimuler et dirigerl'employé qui a cet esprit pour qu'il puisse développer sa personnalité et contribuer à l'expansion de la firme.

Les résultats de cette recherche confirment le fait que la motivation et la productivité des employés étaient des préoccupations stimulantes pour les entrepreneurs dans les premières années de leur entreprise².

2 Résultat moyen de 1,9 sur une échelle de 1 à 5, 1 étant extrêmement important. 
Comment a-t-on pris en main les questions reliées à la motivation des employés? Cette recherche a mis en évidence quatre importants moyens ou méthodes pour favoriser l'esprit entrepreneurial des employés :

1 Près de 77\% des sondés ont mentionné offrir «un environnement propice à la créativité», «peu de règlements», «la possibilité pour l'employé de se créer un travail stimulant», et «un encouragement à l'implication dans leur travail». Près de $24 \%$ des personnes interrogées ont signalé qu'elles «traitaient leurs employés équitablement» et «en adultes». Ces données semblent corroborer une conclusion d'un autre rapport selon lequel nombre d'entrepreneurs se sentaient frustrés et étouffés dans leur profession ou emploi précédent et qu'ils voulaient fonder une entreprise pour laquelle ils aimeraient travailler (Collins et Moore, 1964).

2 Exactement $50 \%$ des personnes qui ont répondu ont dit que «la possibilité d'une participation dans l'entreprise ou des primes de rendement» étaient de puissants moyens de motivation. De même, près de $27 \%$ des sondés offrent «d'excellents programmes de rémunération, des avantages sociaux et d'autres bénéfices financiers». Dans quelques cas, l'entrepreneur a suggéré l'embauche d'un candidat plus qualifié que ne l'exigeait le poste à combler.

3 Près de $27 \%$ des personnes interrogées ont indiqué que l'élaboration d'objectifs personnels et d'entreprise dans le cadre d'un travail d'équipe était un excellent moyen de motivation. Près de $21 \%$ des entrepreneurs ont également noté l'importance de «la reconnaissance publique d'excellents résultats obtenus au travail».

4 Près de $18 \%$ des sondés ont indiqué avoir utilisé le fait que leur entreprise était «un futur leader de l'industrie» pour motiver leurs employés. Cela semble indiquer que la possibilité «de mettre la main à la pâte dès les débuts de l'entreprise» motive l'employé à s'impliquer dans la petite entreprise et à devenir un élément important de la réussite de la firme.

\subsubsection{La délégation des responsabilités}

Certains entrepreneurs n'ont eu aucune peine à déléguer des responsabilités à leurs employés tandis que d'autres ont trouvé très difficile de le faire, particulièrement dans les débuts de leur entreprise, quand ils avaient l'impression qu'eux seuls pouvaient faire le travail correctement.

- $41 \%$ d'entre eux ont indiqué que l'assignation des responsabilités dépendait du volume de travail et de leur confiance en leurs employés.

- $30 \%$ ont dit que la délégation des responsabilités était difficile avant que l'employé ne connaisse son travail et ne soit productif, mais ils ont généralement délégué des responsabilités plus tôt que prévu à cause de leur surcharge de travail.

- $18 \%$ ont répondu qu'ils pouvaient s'occuper de tout pendant les premières années de leur entreprise. 
- $18 \%$ ont dit qu'ils laissaient «carte blanche» à leurs employés et que déléguer faisait partie de leur style de management.

- $18 \%$ ont confié qu'assigner des responsabilités était très difficile.

Les entrepreneurs ont quelquefois appris à céder leurs responsabilités en les confiant graduellement à des subordonnés. Ce cheminement a été très méthodique et prudent et il était frustrant pour eux de devoir accepter les erreurs des autres. Ils ont aussi appris à déléguer à partir de leur expérience de travail passée, en discutant avec d'autres personnes et en suivant des cours.

\subsubsection{La rémunération}

La plupart des entreprises sondées offrent à leurs employés les régimes d'intéressement suivants :

- Participation aux bénéfices pour tous les employés $(71,4 \%)$

- Primes d'encouragement sous forme d'actions pour les employés occupant des postes-clés $(32,4 \%)$

- Primes de rendement pour tous les employés (30\%)

- Primes de rendement pour les employés occupant des postes-clés $(26,7 \%)$

\subsection{Comment la croissance de l'entreprise a-t-elle influencé la structure organisationnelle?}

Le tableau 2 (p. 20) permet de bien visualiser l'évolution du chiffre d'affaires des entreprises considérées. Le tableau 3 (p. 20) présente les nombres médians d'employés, de personnes qui relèvent du P.D.G., le nombre moyen de niveaux organisationnels et le nombre médian de membres du personnel qui ont quitté l'entreprise (pour quelque raison que ce soit).

\subsubsection{Observations}

\section{Ventes}

Le volume de ventes des entreprises manufacturières (SIC's 20-25, 26-29) semble présenter une plus forte croissance annuelle que ceux des firmes des autres secteurs industriels. (En cinq ans, les firmes du secteur manufacturier ont multiplié par 7 leurs ventes de la première année par rapport à 4 et 5 respectivement pour le secteur des services et celui de la vente de gros et détail.)

\section{Le nombre d'employés}

Les entreprises manufacturières montrent une tendance de croissance plus élevée de l'entreprise et du nombre d'employés de la première année à aujourd'hui.

Les entreprises dirigées par des gens qui possèdent une expérience dans le domaine technique ou en ingénierie et de l'expérience de gestion indiquent une 
Tab. 2 Volume de ventes annuel moyen (en milliers de \$)

\begin{tabular}{|c|c|c|c|c|c|c|}
\hline & Année 1 & $\begin{array}{c}\text { Année 5- } \\
X \text { fois } \\
\text { Année } 5\end{array}$ & Année 1 & Année 10 & Aujour & $\begin{array}{l}\text { Age } \\
\text { moyen }\end{array}$ \\
\hline $\begin{array}{l}\text { Ensemble des } \\
\text { entreprises (n-44) }\end{array}$ & 465 & 3064 & $6 x$ & 7923 & 19316 & 14,3 \\
\hline $\begin{array}{l}\text { Entreprises } \\
\text { manufacturières }(n-25)\end{array}$ & 563 & 3638 & $7 x$ & 5895 & 12196 & 12,9 \\
\hline $\begin{array}{l}\text { Entreprises } \\
\text { gros et détail (n-7) }\end{array}$ & 250 & 1125 & $5 x$ & 1929 & $33300^{\prime}$ & 22,1 \\
\hline $\begin{array}{l}\text { Entreprises de } \\
\text { services (n-12) }\end{array}$ & 386 & 1639 & $4 X$ & 7429 & 12583 & 10,8 \\
\hline $\begin{array}{l}\text { Entrepreneurs avec expé- } \\
\text { rience de gestion (n-24) } \\
\text { Entrepreneurs avec expé- } \\
\text { riences tochniques et expé- } \\
\text { riences de gestion }(n-20)\end{array}$ & 448 & 3625 & $7 x$ & 8536 & 18677 & 14,4 \\
\hline
\end{tabular}

- Chiffres estimés à partir de la valeur moyenne de l'intervalle d'une échelle de revenu indiqué par les sondés

1 Ce chiffre est très élevé parce que les entreprises de gros et détail ont en moyenne 22 ans comparativement à 14 ans pour les autres entreprises.

Tab. 3 (Données en valeur moyenne')

\begin{tabular}{|l|c|c|c|}
\cline { 2 - 4 } \multicolumn{1}{c|}{} & Année 1 & Année 5 & Aujourd'hui \\
\hline $\begin{array}{l}\text { Nombre d'employés (n-40) } \\
\text { Nombre d'employés relevant } \\
\text { du directeur-général }(n-46)\end{array}$ & 7 & 19 & 34 \\
Niveaux hiérarchiques $(n-43)$ & 2 & 8 & 8 \\
Employés ayant quitté $(n-31)$ & 1,8 & 2,5 & 3,7 \\
\hline
\end{tabular}

- chiffres basés sur 51 entreprises consultées. 
tendance de croissance plus rapide et ont embauché plus d'employés de la première année à aujourd'hui.

Le nombre d'employés et la croissance des firmes en exploitation depuis 1976 avaient tendance à être plus élevés pendant les cinq premières années d'exploitation que pour les entreprises lancées avant 1976.

\section{Les employés qui relèvent du directeur général}

Le directeur général d'une entreprise indépendante nouvellement lancée avait généralement sous ses ordres 3 ou 4 employés au cours de la première année d'exploitation. Dans les entreprises de service (SIC's 46-49, 60-67, 70-89), le plus souvent, seulement 2 ou 3 employés relevaient directement du P.D.G. pour la même période.

Après cinq ans d'exploitation, le directeur général d'une entreprise lancée individuellement avait, dans la plupart des cas, entre 6 et 8 employés sous ses ordres.

Dans le secteur des services et dans le cas des entreprises fondées depuis 1976, il n'en comptait plus souvent que 4 ou 5.

En 1986, pour beaucoup d'entreprises, la courbe de croissance du nombre moyen d'employés relevant du directeur général s'était stabilisée ou avait décliné légèrement.

\section{La rotation du personnel}

Généralement, trois employés sur dix (en moyenne) ont quitté leur entreprise, volontairement ou non, au cours de sa première année d'exploitation. Le taux de rotation de la main-d'oeuvre, pendant la première année d'exploitation, semble plus élevé dans les entreprises fondées depuis 1976 et dans celles dirigées par des gens ayant une expérience technique et en ingénierie en plus de l'expérience de gestion.

Après cinq ans d'exploitation, les entreprises dont le dirigeant n'a qu'une expérience de gestion perdaient moins d'employés (2 sur 16) que celles dirigées par l'autre type d'entrepreneur ( 10 sur 22 , presque $50 \%$ de leurs employés). Il existe trois explications possibles de ce phénomène : a) il est peut-être plus difficile d'établir de bonnes relations de travail avec l'entrepreneur ayant une expérience technique; b) le secteur d'industrie dans lequel opère un entrepreneur technicien se prête davantage aux changements d'emplois ou donne même aux employés l'occasion de partir et de fonder leur propre entreprise; c) l'entrepreneur expérimenté en gestion seulement met l'accent, quant à lui, sur les systèmes de gestion qui, en retour assurent une plus grande stabilité et un faible taux de roulement du personnel.

En 1986, les entreprises du secteur manufacturier de l'échantillon avaient un taux de rotation de la main-d'oeuvre de $20 \%$, tandis qu'il s'élevaità près de $50 \%$ dans les secteurs de services et de commerce de gros et détail. Comme on s'y attendait, le 
secteur manufacturier présente une plus grande stabilité de l'emploi que celui des services ou de la vente. Toutefois les taux de rotation du personnel semblent quand même élevés dans tous les secteurs.

\section{La complexité organisationnelle}

Pendant la première année d'exploitation, l'organigramme des entreprises comptait généralement un ou deux niveaux hiérarchiques. A la cinquième année, il avait évolué de façon différente :

$\checkmark$ Les entreprises de fabrication se structuraient en trois ou quatre niveaux hiérarchiques, tandis que les firmes de services et de commerce de gros et détail présentaient habituellement une structure à un ou deux niveaux.

$\checkmark$ En moyenne, l'organigramme des entreprises fondées depuis 1976 comptait maintenant trois ou quatre niveaux et celui des firmes plus vieilles en avait deux.

\subsection{A quelles étapes du processus d'embauche d'un candidat, une aide pourrait étre apportée à l'entrepreneur ?}

Le tableau 4 présente les étapes du processus d'embauche d'un candidat où les entrepreneurs perçoivent qu'une aide leur serait utile. Etant donné que les entrepreneurs ne jugent pas essentiel d'étudier un très grand nombre de curriculum vitae, il était manifeste qu'ils montreraient un immense intérêt à être assistés dans la recherche de candidats et à la présélection de leur curriculum vitae. Les dirigeants des firmes plus jeunes étaient intéressés à recevoir de l'aide pour tester les candidats $(1,7$ sur l'échelle), tandis que l'assistance pour les entrevues prenait le deuxième rang en importance pour les entrepreneurs du secteur des services (2,1 sur l'échelle).

Tab. 4 Etapes du processus de sélection

(où une aide serait utile à l'entrepreneur)"

\begin{tabular}{|l|c|}
\hline Étapes & $\begin{array}{c}\text { Importance accordée par } \\
\text { ''entrepreneur' }\end{array}$ \\
\hline 1 Pré-sélection des curriculum vitae & 1,7 \\
2 Recherche de candidats & 1,8 \\
3 Vérification des références & 2,2 \\
4 Test & 2,2 \\
5 Entrevues & 2,6 \\
6 Révision des curriculum vitae & 3,3 \\
7 Identification des conditions requises & 3,0 \\
pour un nouveau poste & 3,2 \\
9 Description des tâches & 3,5 \\
\hline
\end{tabular}

- Echelle croissante de 1 à $5 ; 1$ représentant de grandes économies de temps et d'argent et 5 aucune économie.

1 Sur une base de 42 entrepreneurs consultés. 


\section{Conclusion}

Cette recherche démontre que les pratiques de gestion des ressources humaines d'une entreprise qui démarre sont multiples et complexes et qu'elles diffèrent selon la compétence de l'entrepreneur, le secteur de l'industrie et la croissance de la firme. De plus, l'environnement social, culturel et économique dans lequel est lancée l'entreprise joue également un rôle.

Le recrutement efficace de ressources humaines dans l'entreprise indépendante doit concilier les éléments suivants : 1) la culture et les ressources de l'organisation; 2) les postes, les fonctions et l'autorité; 3) la compétence, l'expérience et la personnalité de gens. La grande entreprise, beaucoup plus stable, n'est pas touchée par certains des problèmes complexes de recrutement qui se posent à l'entreprise entrepreneuriale. Cette demière prend naissance dans l'incertitude (i.e. survivra-t-elle? A l'aide de quelles ressources ?) et nécessite l'accomplissement de tâches complexes, changeantes et stressantes (Kets de Vries, 1984). De même, dans les premiers moments de l'existence de la firme, les valeurs, la compétence et le comportement interpersonnel de l'entrepreneur auront un impact fondamental sur la dimension organisationnelle de l'entreprise, ainsi que sur les tâches de chacun des employés.

Il ressort de cette étude que les entrepreneurs abordent le processus d'embauche avec une certaine ambivalence. Il semble qu'ils engagent, dans les débuts de l'entreprise, du personnel pour deux raisons :

1 pour se décharger d'une partie de leurs responsabilités et ainsi pouvoir travailler sur des problèmes plus urgents,

2 pour occuper un poste à combler qu'ils ne peuvent assumer eux-mêmes à cause d'un manque de qualifications.

En outre, l'entrepreneur s'empresse souvent d'engager une personne pour répondre à des besoins pressants ou courants. Il risque ainsi de négliger de prendre en considération (ou décide d'ignorer) les conséquences à long terme de ses décisions (par exemple, la compatibilité de l'employé à son style personnel ou sa capacité d'adaptation à la croissance de l'entreprise).

Le manque de prévoyance peut entraîner l'embauche ou la promotion d'une personne inapte à occuper un poste (par exemple, un ancien et fidèle employé qui n'a pas les qualifications et le leadership nécessaires pour satisfaire aux besoins d'une entreprise en expansion) avec pour conséquences des départs, volontaires ou non, souvent vécus péniblement. Il est intéressant de noter que l'entrepreneur ne semble pas très occupé par les départs des employés (même s'il y en a beaucoup), et que le poste de directeur des ressources humaines est perçu comme le dernier en importance à combler malgré que d'autres recherches aient souligné que cette fonction, de même que le recrutement en prévision des besoins futurs, soient des éléments essentiels à la croissance de l'entreprise (Hambrick et Crozier, 1984). 
Cette étude constate que les premiers employés doivent être polyvalents (des hommes-orchestres) et doivent posséder de l'expérience, de préférence dans la petite entreprise, alors qu'on a tendance à confier aux employés engagés ultérieurement des tâches de plus en plus spécialisées (ce qui peut provoquer des frictions entre les employés de la première heure et les nouveaux arrivants). Quoiqu'ils s'attendent à ce que les premiers employés soient généralistes, les entrepreneurs semble-t-il, en particulier ceux qui ont une expérience technique, sont enclins à s'entourer d'une équipe «à leur image», composée de personnes ayant la même expérience et qui partagent les mêmes valeurs ce qui, bien que sécurisant, peut constituer un obstacle majeur à la croissance de l'entreprise (Bird, 1990).

Cette enquête soulève certaines différences entre les firmes, selon leur âge. Elles pourraient être attribuables au fait que les entreprises lancées dans les années soixante évoluaient dans un contexte socio-économique différent de celui des entreprises lancées dans les années quatre-vingt; ou encore, à la mémoire sélective ou défaillante des fondateurs des plus anciennes firmes.

\subsection{Conclusions additionnelles}

1 L'entrepreneur doit donner des responsabilités aux employés pour qu'ils deviennent eux-mêmes des entrepreneurs. Il doit leur permettre de faire preuve de créativité, de prendre des risques tout en leur laissant la paternité et la fierté de leurs réalisations.

2 La participation aux bénéfices de l'entreprise et les primes de rendement développent la productivité et le sens des responsabilités des employés.

3 Les erreurs de recrutement et l'absence d'un directeur de ressources humaines, un poste auquel l'entrepreneur accorde peu d'importance, pourraient expliquer le haut taux de rotation du personnel dans les entreprises sondées.

4 Un service d'aide sans but lucratif peut être établi pour assister l'entrepreneur dans le recrutement de départ.

\section{Annexe}

Serait-il possible de mettre sur pied un service d'aide aux nouveaux entrepreneurs pour le recrutement d'intéressants candidats à des postes administratifs ?

L'interprétation des réponses à cette question ne permet pas de tirer des conclusions. Toutefois, nous pouvons envisager la situation avec optimisme, car plus de $50 \%$ des personnes interrogées ont dit qu'elles utiliseraient un tel service (26 oui, 23 non). Il ne semble pas se dessiner un segment de marché spécifique à exploiter puisque différents types d'entrepreneurs de diverses industries ont pris, dans une proportion égale, des positions opposées sur la question. Les entreprises créées au cours des dix dernières années étaient plus ouvertes au concept (15 pour et 3 contre) ce qui est un élément positif. 
Sur une échelle de 1 à 5 , un tel service sans but lucratif a obtenu une cote de 2,4 , ce qui tend à le situer parmi les services intéressants. Cependant ceci $n$ 'est pas une réponse très favorable. Malgré tout, la plupart des personnes consultées nous ont fait des remarques encourageantes :

- $38 \%$ ont mentionné que ce service permettrait des économies de temps pour les entreprises qui démarrent.

- $32 \%$ ont dit que ce service pourrait être utile parce que les nouveaux entrepreneurs n'ont pas de relations dans le milieu.

- $\quad 19 \%$ ont dit que ce service devrait être peu coûteux, car les entreprises nouvellement formées n'ont pas beaucoup de fonds à allouer à la recherche de candidats.

- $16 \%$ ont encouragé la mise sur pied d'un service qui pourrait garder une liste de candidats qualifiés pour travailler dans la petite entreprise.

\section{Autres commentaires :}

- La petite entreprise en expansion a plus besoin d' «indiens» que de «chefs».

- «Soyez prudents, vous ferez concurrence aux entreprises spécialisées dans le recrutement.»

- $8 \%$ croient que les gestionnaires devraient provenir du sein même de l'entreprise.

Nous planifions actuellement la mise sur pied d'un programme de recrutement pour les petites et jeunes entreprises (celles qui ont un chiffre d'affaires de moins de 10 millions). Il sera constitué d'un logiciel et d'une base de données à partir desquels seront appareillés les renseignements contenus dans les curriculum vitae d'employés potentiels et les descriptions de tâches relatives à des emplois disponibles.

\section{BIBLIOGRAPHIE}

Bird, B.J., (1990), Entrepreneurial Behavior, Boston, MA : Littel Brown, (à paraître).

Collins, O.F. et Moore, D.G., (1964), The Enterprise Man, East Lansing, MI : MSU Business Studies.

Hambrick, D.C. et Crozier,L.M., (1984), «Stumblers and stars in the management of rapid growth», Journal of Business Venturing, 1, (1), pp. 31-45.

Kets de Vries, M.F.R., (1984), «Can you survive as entrepreneur?», Cambridge,MA : Harvard Business School Teaching Note 9-4848081.

Lewis, J.L., Sewell, C.H. et Dickson, C.L., (1961), Identification and Evaluation of Problems and Needs of Small Manufacturing Management, Atlanta, GA : Georgia Institute of Technology.

Martin, M.J.C., (1984), Managing Technological Innovation and Entrepreneurship, Reston, VA : Reston Publishing.

Vesper, K.H. (1980), New Venture Strategies, Englewood Cliffs, NJ : Prentice-Hall. 